\title{
Nucleic Acid Sequencing
}

National Cancer Institute

\section{Source}

National Cancer Institute. Nucleic Acid Sequencing. NCI Thesaurus. Code C18881.

The process of determining the sequence of purines and pyrimidines in nucleic acids and polynucleotides. 